\title{
Review of: "Global dissemination of tet(X3) and tet(X6) among livestock-associated Acinetobacter is sporadic and mediated by highly diverse plasmidomes"
}

\author{
Gabriel Trueba ${ }^{1}$ \\ 1 Universidad San Francisco de Quito
}

Potential competing interests: The author(s) declared that no potential competing interests exist.

The manuscript "Global dissemination of tet(X3) and tet(X6) among livestock-associated Acinetobacter is sporadic and mediated by highly diverse plasmidomes" describe the isolation of Acetinobacter spp. from different sources and characterization of tet $(X)$ genes (conferring resistance to tigecycline) and the genetic environment (plasmids, transposons and other genes) associated with tet(X3) and tet(X6).

The authors isolated 684 strains of Acinetobacter spp. from different farms and PCR analysis showed that 9 isolates carryed tet(X3) and 5 isolates carried tet(X6). This indicate that tet(X) carriage is rare in the environment. The majority $(n=10)$ of tet(X)strains were clonal; ( 8 carrying tet(X3) and 2 carrying tet(6)) and all clonal strains carried similar plasmids. Most of these strains failed to conjugate (only one strain was able to transfer the plasmid to other Acinetobacer strain). This is an evidence that horizontal gene transfer of these genes is infrequent and dissemination of tet $(X)$ genes in Acinetobacter occurs by clonal dissemination. This finding agrees with previous publications.

The plasmids detected in this study showed little homology with other plasmids carrying tet(X) genes in the genbank. Nevertheless all these plasmids shared regions of homology specially in the region coding for tet $(X)$ genes. The modules containing Tet $(X)$ genes shared the same transposable elements (such as ISCR2, Tn3, IS6, etc) which suggests that tet(X) genes are jumping (transposing) among different plasmids. This finding agrees with previous publications.

The main problem with this manuscript it that the authors fail to interpret their results adequately. Their interpretation is that tet $(X)$ positive strains "disseminate sporadically" in farms. They don't discuss whether clonal expansion or horizontal gene transfer are important in the dissemination of tet(X) genes. Also, it seems like the authors are wrong about the role of ISCR2 in gene dissemination which is to capture genes and transpose them from chromosomes (or plasmids) to conjugative plasmids. This element is not conjugative per se.

They also conclude that plasmids carrying tet $(X)$ genes in Acinetobacter are very diverse which is an incomplete description of what is going on. 
